\title{
LES CÉDÉROMS GRAND PUBLIC DE CULTURE SCIENTIFIQUE : IMAGES ET REPRÉSENTATIONS DU SAVOIR
}

\author{
Anthippi Potolia'
}

Les cédéroms grand public sont venus depuis quelques années élargir la catégorie des médias de diffusion des savoirs non formels. De nature hétérogène, ces supports font appel à une diversité de codages dans la mise en scène du savoir, à une multiplicité de voix énonciatrices et multiplient, pour l'utilisateur, les opérations de traitement cognitif. Cette pluralité du cédérom est certes à mettre en rapport avec la complexité sémiotique du multimédia proprement dit, mais aussi avec les conditions particulières de production et de diffusion du savoir dans les titres en question.

Avant de nous référer de manière plus spécifique au corpus que nous avons sélectionné dans le cadre du présent article, nous aimerions aborder la question de l'approche conceptuelle à laquelle les supports multimédias semblent mieux se prêter. Différents chercheurs, que ce soit en analyse du discours ou en psycholinguistique textuelle s'accordent aujourd'hui pour affirmer que "la diversification des techniques d'enregistrement et de restitution de l'image et du son est en train de modifier considérablement la représentation

\footnotetext{
1 École Normale Supérieure Lettres et Sciences Humaines.
}

Recherches en communication, $\mathrm{n}^{\circ} 15$ (2001). 
traditionnelle du texte"l et que l'on peut d'ores et déjà inclure, entre autres, dans l'ensemble texte, "un logiciel sur une disquette, un mélange de signes verbaux, musicaux et d'images sur cédérom"2. Il s'ensuit donc que ces derniers supports, de par le statut textuel qui leur est, à juste titre, conféré, remettent en question les outils méthodologiques, élaborés tant par l'analyse du discours que par la linguistique textuelle: l'hétérogénéité sémiotique ne peut plus aborder l'image dans ses rapports de simple transcodage ou même, pour certains, de parasitage du texte ; la cohésion, en termes de superstructure, n'est plus à considérer du point de vue de l'articulation logique ou rhétorique des connecteurs; le paratexte dans sa dimension scripto-visuelle évolue également, non seulement sur le plan transémiotique, mais aussi dans sa capacité de créer du sens. Il se transforme souvent en une condition sine qua non pour entrer dans le sens.

Ainsi, il nous semble qu'il y a toutes sortes de correspondances à établir entre le multimédia hors ligne et la réflexion menée autour du musée ou de l'exposition en tant que mise en espace produisant du langage ${ }^{3}$; mise en espace globale, multipliée ou déclinée à son tour en plusieurs sous-espaces ayant chacun sa propre scénographie non seulement discursive mais aussi sémiotique ${ }^{4}$; sous-espaces qui "s'apparentent à" mais "sans être réellement". Ainsi, de par leur rattachement à l'espace global et leur nature mimétique, ils participent, chacun à leur façon, à la construction de l'image du savoir ; savoir qui, derrière son iconicité plastique, figurative, discursive, révèle un deuxième niveau de sens qui a partie liée avec sa représentation, auprès du grand public.

Mais il y a aussi un autre point : les cédéroms tels que nous venons de les définir dans leurs grandes caractéristiques sont produits et mis en circulation par des espaces à dominante médiatique "organisant un marché des textes"s. Ils sont ainsi rattachés à un

1 D. Maingueneau, Analyser les textes de communication, Paris, Dunod, 1998, p. 43.

2 P. CoIrier, D. GaOnaC'H, J.-M. Passerault, Psycholinguistique textuelle: une approche cognitive de la compréhension et de la production des textes, Paris, Armand Colin, 1996, p. 7.

3 J. DAVALlon, L'exposition à l'auvre, Paris, L'Harmattan, 1999, p. 18.

4 D. MAINGUENEAU op. cit., pp. 70-76.

5 J.-C. BEACCO, "Cadre théorique de l'analyse : les communautés discursives", $L$ 'astronomie dans les médias, Analyses linguistiques de discours de vulgarisation, Paris, Presses de la Sorbonne Nouvelle, 1999, p. 15. 
premier interdiscours qui a, entre autres, favorisé leur avènement en tant que médias, puis façonné leur écriture dans ses grandes caractéristiques (et qui continue d'ailleurs à le faire, puisqu'il est ici question d'une écriture non encore stabilisée ou qui n'a peut-être pas vocation à l'être). Mais dans le cadre du corpus que nous examinerons ici, il y a aussi la voix de la science qui renvoie à un deuxième interdiscours, avec ses propres enjeux, son propre continuum communicationnel (informel, institutionnel, public) ${ }^{1}$. Ainsi un cédérom en tant que produit fini, tel qu'il est donné à voir au terme du "lieu de construction du discours", ne peut pas être envisagé comme un simple contenu informationnel. Il porte au contraire en lui, les traces des représentations qui ont nourri la réflexion de l'instance émettrice et qui ont guidé ses choix. Ces représentations (du public-cible, de la science et de son image auprès du grand public, du cédérom en tant que support de consultation personnelle et personnalisée, vecteur de connaissances et d'émotions, etc.), forgées dans les deux types d'interdiscours auxquels nous nous sommes référée supra, influent sur la structure, la scénographie, l'éthos de chaque titre et ne sont repérables qu'à travers les images privilégiées par ces interdiscours.

Pour revenir au moment présent et tâcher d'illustrer et éclaircir ces quelques ancrages théoriques, précisons que nous nous limiterons ici à quelques-unes des caractéristiques de deux cédéroms émanant de la même instance éditoriale. Il s'agit des titres : Aux origines de l'homme (1994, réédité en 1996) et L'océan des origines (1996). Ils se rattachent tous les deux à la paléontologie, déclinée en deux sousdisciplines distinctes: la première s'intéresse à l'homme et à ses origines (paléoanthropologie); la deuxième aux fossiles marins et à la vie des océans ${ }^{3}$. Bien que leur objet de prédilection soit différent, elles usent toutes les deux d'opérations de reconnaissance, de reconstitution, de comparaison, de localisation dans l'espace-temps et elles sont sur le plan discursif cernées par le même doute vis-à-vis de la validité de leurs interprétations ou de leurs thèses et par une même remise en question continuelle de leurs démarches.

1 J.-M. Levy-Leblond, La Pierre de touche, Paris, Gallimard, 1996, p. 235.

2 P. Charaudeau, Le discours d'information médiatique, Paris, Nathan, 1997, pp. 18-20.

3 Pour des raisons de commodité nous parlerons ici de paléoanthropologie pour désigner la discipline représentée par Aux origines de l'homme et de paléontologie dans le cas de L'océan des origines. 
Nous chercherons, dans le cadre de cette contribution, à mettre en avant les convergences dans la présentation du savoir au sein des deux cédéroms retenus pour l'analyse, puis les divergences repérées dans leur mise en discours et leur mise en texte. Cela nous permettra de dégager, dans un premier temps, l'évolution de l'image du cédérom en tant que support textuel au sein de la même instance éditoriale ; et de voir, dans un second temps, de quelles façons deux disciplines différentes s'appuient sur un même support pour transmettre l'image d'un savoir qu'elles privilégient ou qu'elles pensent être celle recherchée par le grand public.

\section{Quelques éléments d'analyse}

Il nous semble, avant tout autre chose, opportun de présenter la géographie des deux titres étudiés dans ses grandes lignes et ce, afin de faciliter la lecture de l'analyse que nous proposerons dans la suite de notre propos.

Le menu principal du cédérom Aux origines de l'homme, se présente sous la forme d'un bureau sur lequel se trouvent éparpillés différents documents donnant, pour chacun d'entre eux (et une fois activés), accès à un espace de savoir différent. Si certains de ces espaces -tels que les entrées Bibliographie, Index, Testez vos connaissances- nous préparent d'emblée à ce que seront leurs propriétés textuelles, la plupart d'entre eux ne nous rendent compte de leur format discursif et scénographique qu'une fois activés. Ainsi, dans l'Échelle du temps, un diaporama, suivi d'une voix off présente les conditions climatiques qui ont favorisé, sur une période de soixantedix millions d'années, le développement de certains hominidés ou qui ont, au contraire, contribué à la disparition de certains autres; les Chemins de l'évolution proposent un arbre phylogénétique qui montre les filiations entre les espèces depuis l'apparition de la vie. Un bref commentaire écrit pour chaque espèce, la présente dans ses grandes caractéristiques (taille, caractéristiques morphologiques, habitudes alimentaires, etc.) ; l'entrée Ancêtres et cousins, nous permet d'étudier de près dix-huit de nos ancêtres et cousins (ancêtres de certaines espèces de singes actuels). Nous pouvons voir et entendre les différents primates, les comparer entre-eux, avoir un aperçu global sur la faune de leur époque ; l'entrée Transformations utilise la technique du morphing pour présenter les différents primates en 3D; dans les 
Découvertes paléontologiques deux personnes en voix off et devant un album de photos commentent quelques-unes des plus importantes découvertes de primates; sous la forme d'un vieux livre, l'entrée Théories de l'évolution relate les théories concernant l'origine et l'évolution de l'Homme; pour terminer, l'espace Une expédition archéologique, permet à l'utilisateur de découvrir sous forme de simulation les grandes étapes d'une expédition, les méthodes utilisées, les différents spécialistes qui y participent et leur fonction.

\section{Aux origines de l'homme, Menu principal}

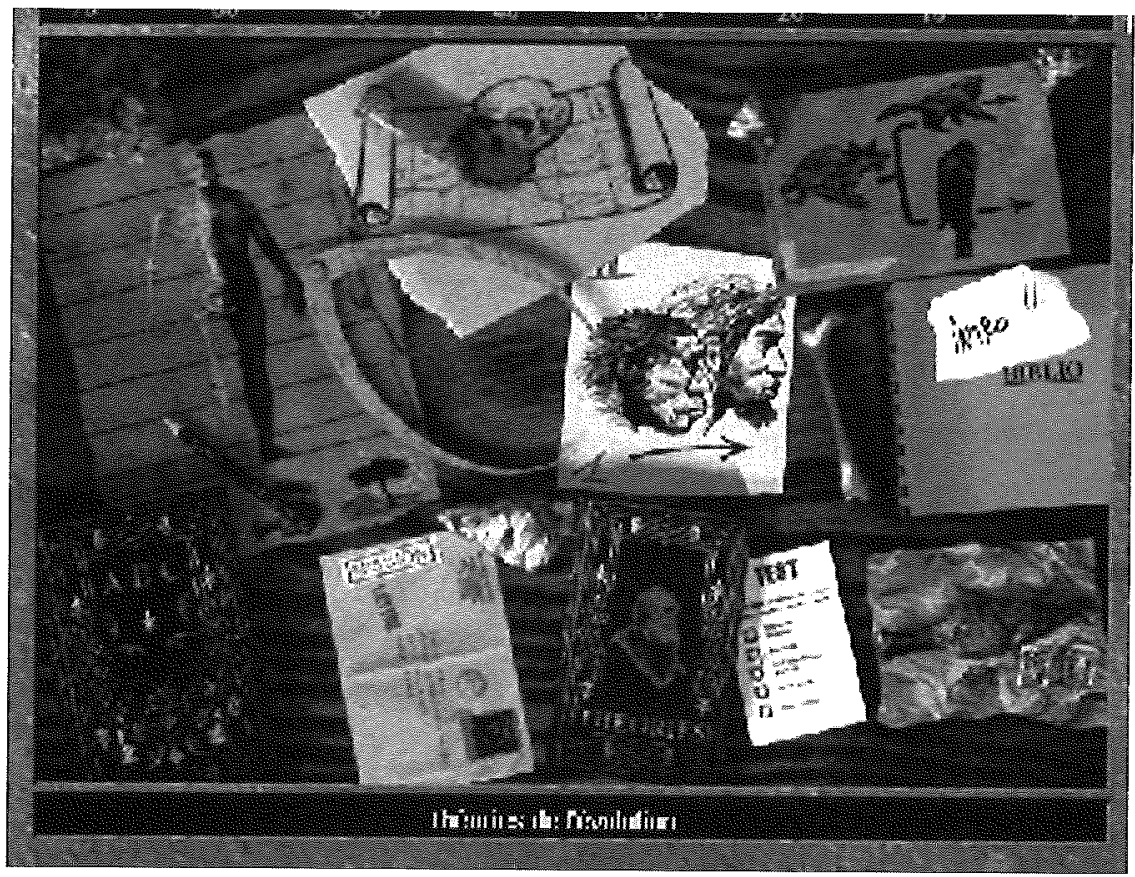

(C) Microfolie's 1994 et 1996

Le menu principal de L'océan des origines représente le hall d'une cité sous-marine. Au centre de ce dispositif se trouve Théthys, la sirène de la cité qui présente l'un après l'autre les différents espaces du savoir dessinés en arrière-plan. Six principales entrées sont ici disponibles, chacune thématiquement reliée au référent global de l'évolution et de l'étude de la vie animale marine : Protéion présente les biographies des savants qui ont fait avancer la connaissance de l'océan ; la Bibliothèque propose un lexique, des fiches sur les différentes espèces animales et végétales, un parcours anatomique permet- 
tant de comparer deux à deux les espèces; Familia vise l'étude de vingt grandes lignées; Fossilis est consacrée aux grands principes de la fossilisation et aux sites de découverte de fossiles; la Salle de projection propose une étude des fonctions vitales ainsi qu'un aquarium virtuel permettant la visualisation des espèces étudiées ; le Paléoscaphe invite à un voyage dans le temps pour découvrir la vie à chaque époque ; un diaporama introductif vient s'ajouter à ces principaux espaces pointant, depuis le Big Bang, toutes les grandes étapes qui ont favorisé le développement de la vie sur la planète Terre. Ces entrées sont conçues comme des univers mimétiques complexes. Une fois activés, ces espaces sont représentés comme des pièces de la cité avec leur propre scénographie selon la sous-thématique à laquelle chacun se rattache. Ici, des objets, des écrans, des appareils divers peuvent être activés pour dévoiler, chacun à sa façon, son savoir. Un savoir présenté tantôt sous forme de jeux, mais qui se déploie le plus souvent par le biais de séquences textuelles à dominante descriptivonarrative, de diaporamas, de reconstitutions virtuelles en 3D.

\section{L'océan des origines, Menu principal}

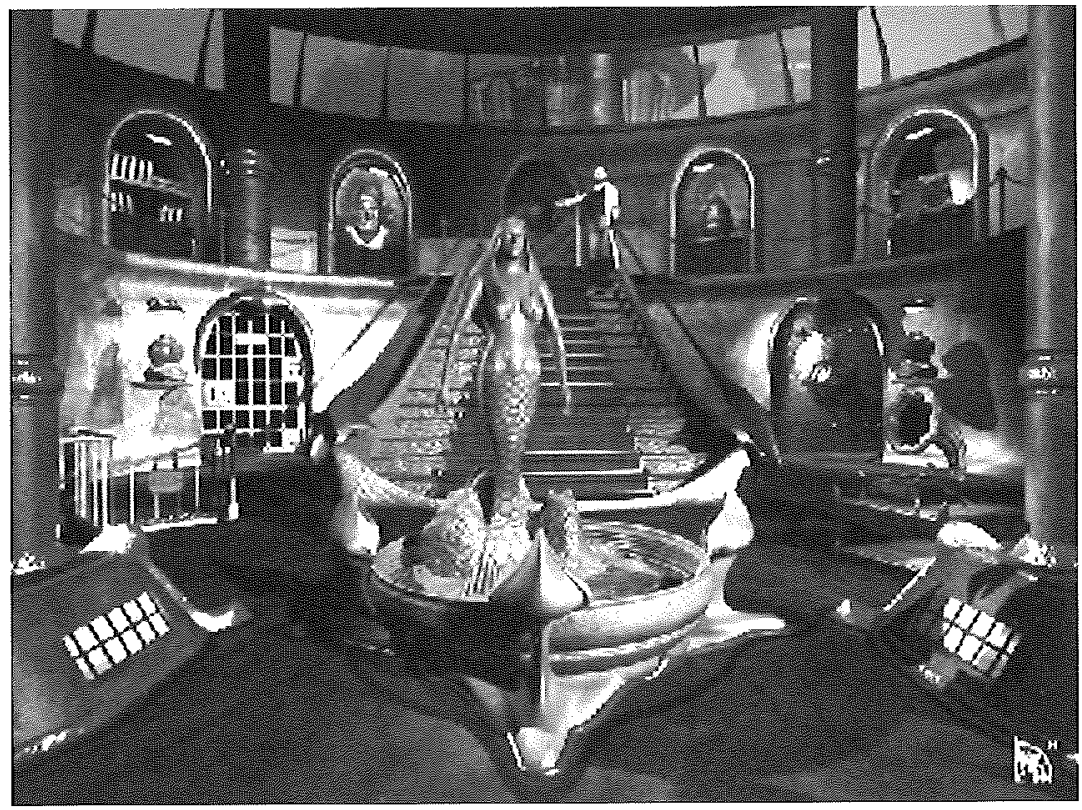

C Microfolie's 1996 


\section{Des traitements communs du savoir}

Nous avons vu supra, et la présentation du contenu de notre corpus le confirme, que le savoir véhiculé par les supports multimédias est de nature plurielle. Mais outre cette pluralité, le savoir en question possède aussi un caractère clos. En effet, le multimédia hors ligne ne peut pas afficher en gros titres une nouvelle découverte; il ne peut pas profiter d'une mise en feuilleton discursive quotidienne, bimensuelle ou même mensuelle. De ce point de vue, il s'apparente au livre, il a fonction de synthèse, d'état des lieux, au risque d'être vite dépassé par les avancées de la science (c'est pour cela d'ailleurs que beaucoup d'ouvrages notamment encyclopédiques, proposent une actualisation en ligne). De ce caractère clos témoigne, la réédition du titre Aux origines de l'homme, réalisée en 1996, deux ans après la première édition, laquelle prend en compte les dernières découvertes paléoanthropologiques, celles d'Australopithecus Anamensis et d'Abel, qui ont respectivement eu lieu en août et en novembre 1995.

Mais outre cet effet de clôture, plus ou moins commun à tout cédérom, le savoir est plus particulièrement défini ici au travers de trois facettes différentes : il est spatialisé, médiatisé sur le plan scientifique, visité dans ses dimensions les plus "humaines".

Sa spatialisation intervient dès les menus principaux qui font office de sommaire. La consultation est ainsi en quelque sorte narrativisée. Dans le cas du cédérom Aux origines de l'homme il est question d'une lecture/navigation qui s'effectue au travers de documents éparpillés sur un bureau. Avec L'océan des origines, la lecture se transforme en visite au sein d'une cité renfermant toute la connaissance sur l'évolution de la vie marine.

La médiation proposée relève de l'ordre du reconnu. Dans le premier cas (Aux origines de l'homme) cette reconnaissance est d'autant plus frappante qu'elle concerne une personne très médiatisée par ailleurs : Yves Coppens. Son titre sur la jaquette du cédérom est long et énumératif: "(...) professeur au Collège de France, découvreur de la célèbre Lucy et auteur de nombreux ouvrages sur la paléontologie". La présence bien plus modeste de Daniel Goujet, collaborateur scientifique à la réalisation de L'océan des origines, justifie en quelque sorte que son nom soit d'abord suivi de sa 
spécialité, "paléontologue", puis, de sa qualité : "professeur au Muséum national d'Histoire naturelle".

Enfin, dans les titres que nous examinons ici, le savoir aborde les objets ou plutôt les sujets qui sont à sa source de la manière la plus humanisée possible. A commencer par les titres mêmes des cédéroms, la notion d'origine revient comme un leitmotiv. L'essentiel est de passer outre l'aspect extérieur, physique des ancêtres de l'homme ou des ancêtres des ancêtres de l'homme pour mettre en exergue ce qui les rapproche le plus de l'utilisateur -l'homme moderne. Cette tâche s'annonce certes plus facile dans le cas du titre Aux origines de l'homme, faisant comme le souligne $\mathrm{J}$. Caune ${ }^{1}$ partie des thèmes qui ont fasciné le public depuis toujours. C'est pour cela d'ailleurs que dans le cas de L'océan des origines le rapprochement entre l'homme et le poisson est beaucoup plus marqué discursivement : ainsi, au niveau du paratexte extra-numérique (le petit livret glissé dans le boîtier du cédérom) nous retrouvons quelques lignes ayant une fonction d'avant-propos, de préambule ou d'accroche :

1a. Sans qu'il s'en rende compte, tout être humain, avant de naître, fait un court séjour dans le mémorial de l'océan des origines. Au stade de foetus, il respire comme un poisson dans le liquide qui le baigne. Son corps en formation porte le souvenir de la première corde qui précéda la colonne vertébrale des vertébrés et ses membres ne sont que des développements des premières nageoires.

Par ailleurs, le diaporama introductif accessible depuis le hall de la cité des océans retrace toutes les grandes étapes de la constitution de la vie qui amènent "jusqu'à nous" :

1b. Parmi toutes les planètes du système solaire/nées il y a QUATRE milliards et demi d'années environ la TERRE est une privilégiée// Son volume ainsi que sa distance par rapport au soleil/ l'étoile-mère/ permet l'existence d'une atmosphère qui garde la vapeur d'eau prisonnière// Sous un ciel irrespirable l'ébauche d'un océan recouvre la planète// La vie commence à se développer dans des eaux privées d'oxygène/ que viennent sans cesse grossir celles qui viennent des météorites ou des éruptions volcaniques// Alors que les continents font surface et commencent à

1 J. CAUNE, La culture en action. De Vilar à Lang : le sens perdu, Grenoble, PUG, 1999, p. 354. 
dériver/algues bleues et bactéries modifient lentement le milieu marin// Il faudra QUATRE milliards d'années pour que l'oxygène qu'elles rejettent donne à l'air sa transparence et à l'océan sa couleur// Une ancienne mer de l'Australie nous a livré des TRACES des premiers animaux à corps mou// Vers/ plumes de mer et méduses/ morts il y a SIX CENT CINQUANTE millions d'années// Puis viendront les luttes pour la conquête du territoire océan// Les espèces se multiplieront// Simples ou complexes elles seront à l'origine de TOUTES les lignées arrivées jusqu'à nous.

Un diaporama similaire est d'ailleurs disponible au lancement du cédérom Aux origines de l'homme qui remonte le temps du Big Bang à Homo sapiens sapiens sans pour autant qu'il soit investi d'un commentaire en voix off.

Mis à part ces passages que l'on peut qualifier d'introductifs en nous référant à notre culture livresque, nous retrouvons tout au long des deux titres une multitude de traces faisant référence à un savoir anthropomorphe : les primates ont un visage et manient adroitement la langue d'aujourd'hui ; ils sont présentés dans leurs habitudes les plus quotidiennes : nourriture, milieu naturel, vie en société, rituels funéraires, etc. De même que les poissons et les animaux marins qui assurent des "fonctions vitales" proches de celles de l'homme, évoluent en passant des nageoires aux pattes leur permettant de "marcher", développent des stratégies d'approche discrète et des facultés de rapidité pour pouvoir "survivre" et ne sont pas présentés seulement dans leur forme fossilisée ou anatomique mais mis en scène dans leur milieu naturel reconstitué en image de synthèse.

Mais ce savoir est aussi humanisé dans la mesure où il reconnaît que ses "découvreurs", à la fois hommes de science et de terrain, constatent, observent, reconstituent, identifient, décrivent mais qui sont aussi passionnés par leur objet de travail, persistants, acharnés, esprits curieux et infatigables.

\section{Des décalages sémio-discursifs}

Malgré ce positionnement global commun vis à vis du savoir, des écarts importants existent dans la mise en espace et la mise en discours de celui-ci. Si l'on transpose au savoir la distinction de Mc 
Luhan ${ }^{1}$ entre les médias "chauds" et les médias "froids" on dira que le savoir paléoanthropologique relève beaucoup plus du "chaud" alors que celui lié à la paléontologie dans sa dimension marine est plus proche du qualificatif "froid". Autrement dit, il est question, dans le premier cas, d'un savoir proche et familier, et, dans le deuxième, d'un savoir mis à une certaine distance.

Ainsi, si nous revenons sur l'exemple des paléoanthropologues et des paléontologues, nous remarquons qu'il existe un décalage très net entre un travail en équipe pour les premiers et un travail solitaire pour les seconds. C'est pour cela, d'ailleurs, que dans les textes se référant aux qualités humaines des chercheurs, celles-ci sont plutôt induites par le discours (implicites) dans le cas de Aux origines de l'homme alors qu'elles sont beaucoup plus marquées linguistiquement (explicites), au travers d'insertions axiologiques, dans L'océan des origines. Comme s'il s'agissait de convaincre, dans ce deuxième cas, le lecteur de la passion et de l'acharnement que l'on peut éprouver vis à vis d'une science qui semble a priori mobiliser beaucoup moins l'imaginaire collectif que la paléoanthropologie.

Par ailleurs, et en ce qui concerne Aux origines de l'homme, nous remarquons des entrées où le savoir, plutôt que de se donner à voir de manière neutre, relevant du descriptivo-narratif, est investi sous forme de monologues ou de dialogues ponctués de marques qui le rapprochent de la langue de tous les jours : des marques d'interaction simulant un face à face avec l'utilisateur (je, me, vous, vos), un lexique et des constructions syntaxiques informels, des appréciations et emplois hypocoristiques, des traits prosodiques vecteurs de sens (accents d'insistance, rapport voix-corpulence, rires, effets exclamatifs $\left.{ }^{2}\right)$ :

2a. Purgatorius : Vous n'en revenez pas ! Une tête pareille/ vous n'y croyez pas ! (...) D'ailleurs la nuit c'est excellent pour la chasse aux insectes//

2b. Necrolemur : Pas question de descendre de mon arbre// J'y suis TROP bien//

2c. Homo erectus : Bon/ j'ai fait beaucoup de progrès depuis mon ancêtre Homo habilis/ ce vieux charognard// (entrée Ancêtres et cousins)

1 M. MC LuHan, Pour comprendre les média, Paris, Mame/Seuil, 1968, pp. 41-52.

2 M.-A. Mochet, "Procédures de reformulation et supports multimédia", Actes du colloque Répétition, altération, reformulation, Annales littéraires de l'Université de Besançon, Presses Universitaires de Franche-Comté, 2000, pp. 239-242. 
3a. $\mathrm{F}$ : Ah dis donc/ ça a l'air sec !

H : Tu parles ! L'équipe de [Van Valen] et [Sloan] avait baptisé l'endroit "la colline du Purgatoire".

F : Ah bon ! Pourquoi ? (...)

$\mathrm{F}$ : (rires) Ah/ oui mais c'est tout petit !

3b. H : Là tu vois/ c'est le Fayum un vrai paradis pour les paléontologues.

F : Ben/ pourtant/ ça n'a pas l'air très vivant. (...) (entrée Découvertes paléontologiques)

De telles situations rapprochent le savoir en paléoanthropologie de ce que J.-C. Beacco ${ }^{1}$ appelle dans le contexte de l'astronomie le "gai savoir" ; savoir dramatisé, animé et image d'une science qui peut pénétrer l'espace quotidien et faire l'objet d'un échange en couple devant un album de photos (cf. l'entrée Découvertes paléontologiques, organisée autour d'un dialogue en voix off entre un homme et une femme, le premier éclairé en la matière, la deuxième ignorante).

Le savoir dans L'océan des origines, est mis à plat, exposé au travers de séquences à dominante narrative et/ou descriptive. La construction syntaxique des énoncés relève du scriptural ou de l'écrit oralisé́ ${ }^{2}$ L'écriture est ici plus soignée, les textes plus longs, l'emploi du passé simple fréquent, le rapport texte-image, beaucoup plus didactique, est en redondance. Des espaces de la cité tels que Fossilis, Familia, Proteïon, de même que l'emprunt du latin pour la dénomination des scientifiques dont les biographies sont présentées dans ce dernier espace, fonctionnent -de par leur nom-comme des interférences diachroniques créant l'image d'un savoir sérieux, axé sur l'intellect. Il en va de même pour l'aspect très cognitif des jeux proposés, traversés par le latin et par une scénographie à polygones et à lignes droites. Une science du détail se dessine alors oscillant entre l'ancien et le nouveau, entre le "lisse" et le "rugueux"3; un savoir "froid" qui doit comme nous l'avons vu, affirmer discursivement ses aspects humanisés tout en les exprimant par une voix autre, ne pouvant pas réellement les faire voir ou les faire entendre par les animaux eux-mêmes :

\footnotetext{
1 J.-C. BEACCo, 'L'actualité des sciences astronomiques dans les quotidiens : le gai savoir", op. cit., p. 199.

2 Cf. à titre indicatif l'exemple $1 \mathrm{~b}$ donné supra.

3 M. Foucault, Les mots et les choses, Paris, Gallimard, coll. “Tel", 1966, p. 144.
} 
4. Au milieu des récifs d'algues au teint violacé les Ajacicyathes se sont ancrés dans le fond peu profond. Leurs formes de bulbe ou de vase les font ressembler à des plantes. Mais ces animaux très simples n'arrêtent pas de pomper l'eau pour survivre. Ils seront supplantés par leurs cousines les éponges aussi parfaitement immobiles qu'eux (...). Les Aysheaias, grimpés dessus à l'aide de leur moignon griffu s'en délectent. Canadaspis arpente les rochers à l'abri de sa carapace glandée. Ottoia s'apprête à rentrer sa trompe avant de s'enfouir dans son trou. Opabinia guidée par ses cinq yeux saisit ses proies à aide d'appendices qui se terminent en pince. Mais ce prédateur peut devenir lui-même la proie du plus grand d'entre eux, le terrible Anomalocaris. Monstres fragiles ou robots merveilleux ces arthropodes primitifs n'ont pas eu de descendance. Est-ce pour cette raison qu'ils continuent de nous étonner ? (entrée Paléoscaphe).

La "gaieté" du savoir dans Aux origines de l'homme ne conteste pas pour autant sa scientificité : on évite l'emploi des dénominations modalisées relevant même parfois du caricatural comme celles que l'on peut trouver parfois dans la presse notamment généraliste ${ }^{1}$; les primates, dans les espaces consacrés à leur comparaison, sont représentés à nu sans recours à un code vestimentaire qui trahirait en quelque sorte l'objectivité des ossements à partir desquels ils semblent avoir été reconstitués. Mais en même temps, cette objectivation et l'anthropomorphisme dont ils font l'objet (n'oublions pas qu'ils sont dotés de la parole) reflètent vraisemblablement, comme le souligne un article du Monde, la tendance actuelle chez les paléoanthropologues qui "s'efforcent de remplacer les effrayants "tableaux vivants" qu'on affectionnait à l'orée du siècle par des reconstitutions plus modestes, mais plus fidèles et rassurantes"2.

Le savoir semble ici plus dynamique et ceci dès le titre même du cédérom. La formulation "Aux origines de l'homme" crée un effet de mouvement, de déplacement, ce qui n'est pas le cas du titre déclaratif "L'océan des origines". Cette dynamique on la retrouve d'ailleurs dans la figuration du menu principal du cédérom. Il s'agit, nous l'avons vu, d'un bureau sur lequel se trouvent éparpillés des

1 D. JACOBI, "«L'Homme» : une nouvelle à caractère scientifique dans la presse et les magazines", Parcours linguistiques de discours spécialisés, Peter Lang, 1993, pp. 10-22.

2 Le Monde, "Cocagne et Cro-Magnon", 27 mars 1993, p. 40. 
documents d'un savoir livresque mais aussi des dessins, des objets faisant référence aux fouilles, des traces d'une mission. La science est donc représentée dans ses aspects d'étude solitaire mais exhibe aussi, même métonymiquement, son goût pour l'aventure. Le menu principal de L'océan des origines, représentant une cité sous-marine, est qualifié dès la jaquette du cédérom comme un espace qui semble surgir "d'un roman de Jules Verne". Cette comparaison induit un savoir certes mystérieux -comme d'ailleurs l'ensemble du dispositif scénique- mais à caractère narrativisé, plutôt que vécu.

\section{Des écarts dans la mise en texte du savoir}

Nous allons maintenant voir comment le cédérom en tant que support médiateur investit l'activité d'accès au savoir de manière beaucoup plus encadrée en passant du premier au deuxième titre. Comme nous l'avons déjà dit, le savoir tant dans le cas de $A u x$ origines de l'homme que dans celui de L'océan des origines est spatialisé à travers les menus principaux de ces deux titres. En replaçant chacun de ces deux cédéroms dans son époque on voit que le premier est édité à l'issue d'une période pendant laquelle les cédéroms sont notamment appréciés dans leur capacité de fonctionner comme des anti-modèles de la lecture des supports "classiques" (idée de navigation dans tous les sens, d'infinité de parcours, d'associations inconcevables auparavant mais aussi, image d'un sujet-lecteur seul "maître à bord" et conscient dans ses choix de construction de sens). Aux origines de l'homme arrive presque en même temps que la collection Triptyques (Arborescence) regroupant sur le même support un peintre, un musicien, un poète et le cédérom consacré aux collections et au palais du Louvre (Montparnasse multimédia). Dans le cas de Triptyques nous retrouvons les caractéristiques d'une lecture trop libre sans presque aucune médiation pour l'articulation cohérente entre les différents espaces. Avec Le Louvre la lecture est spatialisée en ce sens qu'elle s'associe à l'espace physique du musée, extérieur à l'univers mimétique du cédérom. Aux origines de l'homme se présente comme le seul, parmi ces trois cédéroms, à définir explicitement l'espace de lecture, dès son menu principal. Ce procédé sera repris et amélioré dans l'espace du hall de la cité de L'océan des origines. La mise en scène semble, dans ce cas, influencée tant par le succès qu'ont connu les cédéroms de musées que par l'héritage muséal 
propre à la Paléontologie, celui du Muséum national d'Histoire naturelle. Mais s'opère à ce niveau une fracture: Aux origines de l'homme, de par la référence de son menu aux documents écrits, crée l'image d'une lecture a priori encadrée, contrairement à $L$ 'océan des origines où la consultation semble dépendre des déplacements de l'utilisateur dans l'espace. Or, une analyse plus détaillée des deux cédéroms nous montre que dans le premier titre les moyens donnés pour l'accès au savoir renvoient plutôt au "faire voir" alors que dans le deuxième, nous relevons des traces d'un souci de "faire comprendre" ; et ce, tant au niveau de la forme qu'à celui du contenu. Quelques exemples nous permettront de mieux asseoir cette constatation.

Ainsi, dans Aux origines de l'homme le diaporama introductif qui pointe les moments cruciaux de la création de l'univers jusqu'à l'apparition des premiers primates est donné à voir de manière procédurale sans aucun commentaire explicatif en voix off ; l'intitulé des différents espaces accessibles depuis le menu principal est certes affiché en bas de la page-écran mais sans pour autant que leur contenu soit donné de manière plus précise ; il s'agit par ailleurs d'espaces hétérogènes faisant allusion au paratexte livresque (introduction, index, bibliographie) ou pouvant renvoyer à une évaluation (testez vos connaissances), à un jeu (une expédition archéologique), à des contenus informatifs (découvertes paléontologiques, théories de l'évolution $)^{1}$; dans les espaces qui relèvent de l'observation des primates ou de leur comparaison, on met en avant la capacité de l'utilisateur à déceler toutes sortes de décalages entre les primates sans expliquer ou sans lui donner la possibilité de vérifier ses hypothèses ; au niveau du contenu, les chemins de l'évolution sont tracés de manière monophonique passant sous silence les différentes positions des paléoanthropologues dont la presse se fait l'écho ; et, quand le texte exhibe des avis différents, ceux-ci sont plutôt cachés derrière des constructions globalisantes (certains...) sans qu'ils soient présentés plus en détail dans leur argumentaire ; par ailleurs, même lorsqu'on se déplace dans des espaces bâtis a priori autour d'une opération cognitivo-langagière telle que l'explication, il s'agit vraisemblablement de montrer l'explication en train de se construire plutôt que de se soucier de son bon fonctionnement :

1 C. DevelotTe, "Écriture multimédia et nouvelle construction du savoir", Les cahiers de l'Asdifle, Actes des 19e et 20e rencontres, Paris, 1998, p. 40. 
5. H : Dans la première moitié du siècle/ les scientifiques qui croyaient à l'origine africaine de l'homme n'étaient pas nombreux// Robert [Broom] était de ceux-là// Il sillonnait l'Afrique du Sud/ à la recherche de preuves de l'existence des Australopithèques.

$\mathrm{F}:$ Et il en a trouvé ?

$\mathrm{H}:(\ldots)$

$\mathrm{F}: \mathrm{Oh} /$ ben tout ça/ ça m'a l'air bien compliqué.

$\mathrm{H}:$ Ah oui/ et puis ça va pas se simplifier/ (...).

$\mathrm{F}: \mathrm{Mmmm}$ (l'air soucieux)

$\mathrm{H}:(\ldots) / / \mathrm{T}$ as compris?

$F:$ Non

H : Dommage/ c'est pas fini ! (...) (entrée Découvertes paléontologiques).

Inversement, dans L'océan des origines l'arrivée sur le menu principal est précédée d'un commentaire en voix off ancrant la consultation dans l'espace de la cité de l'océan ; une fois dans la cité, Théthys, la guide, présente les différents espaces et le savoir organisé thématiquement à l'intérieur de chacun d'entre eux (Cette démarche d'encadrement est d'ailleurs reprise dans la deuxième édition du cédérom Aux origines de l'homme: un deuxième cédérom vient s'ajouter au premier déjà publié en 1994, dans lequel Y. Coppens lui-même présente et commente les différentes entrées du cédérom principal); un navigateur, sorte de sommaire, est disponible à tout moment en bas de l'écran, donnant à l'utilisateur un aperçu des espaces déjà parcourus lors de sa visite; l'expérience proposée dans l'entrée Fossilis, sorte de jeu de reconnaissance d'un fossile animal, est menée sous le regard bienveillant de Théthys qui informe l'usager de différents procédés dont il dispose pour l'identification des fossiles ; tout au long de la visite on peut faire appel à des fiches présentant les différents animaux du cédérom et les caractéristiques de leur fossile ; même dans le cas des animations, comme l'aquarium virtuel, qui relèvent de l'univers de l'image et qui n'ont pas d'autre prétention que de "faire voir", nous retrouvons des informations telles que la taille réelle des animaux représentés, leur nom, la période géologique pendant laquelle ils ont vécu ; dans le cas d'études comparant les espèces entre elles, plutôt que de se limiter à une mise à plat anatomique, des commentaires sont prévus pointant sur leurs écarts les plus significatifs.

Ces quelques remarques nous ont permis de dégager à côté de l'image de la science présentée dans la section précédente celle du 
support qui en est le vecteur. Ces deux images nous permettront dans une dernière partie synthétique d'émettre un certain nombre d'hypothèses sur les représentations dont elles sont issues : celles que l'instance émettrice (dans sa composante tantôt éditoriale et tantôt scientifique) se fait de son public-cible et de ses attentes d'une science mise sur cédérom, mais aussi d'elle-même.

\section{Quelques éléments de synthèse}

Les cédéroms examinés ici dans leurs grandes lignes visent tous les deux le grand public. Cependant, celui-ci semble être interpellé avec Aux origines de l'homme dans son affectivité alors qu'avec L'océan des origines il serait plutôt question d'un public défini en termes cognitifs. L'émotion, l'usage d'un langage ludique, le non encadrement de la lecture dans le premier cas, vont vraisemblablement de pair avec notre représentation première du cédérom, celle d'un "support-miracle" mettant le savoir en scène de façon différente de celle des autres supports et permettant son acquisition de manière quasi-instantanée. L'objectivité, le sérieux, l'encadrement, en quelque sorte, du regard de l'utilisateur, dans le deuxième cas, correspondent plutôt à une période, où, au fur et à mesure que les réalisations multimédias se multiplient, on partage de plus en plus la thèse qu' "il ne suffit pas d'ouvrir des fenêtres, de parcourir des hyperespaces, de naviguer dans des cédéroms multimédias, de dialoguer avec un programme intelligent"1 pour qu'il y ait appropriation du savoir.

Mais l'opposition affectif-cognitif intervient aussi et surtout au niveau de l'image que chacune des deux sciences s'est forgée lors de sa diffusion auprès du grand public. Il suffit de parcourir les pages d'un quotidien à large diffusion tel que le Monde pour remarquer que derrière la configuration discursive des articles consacrés à la paléoanthropologie se trouve une science vivante, animée, traversée de paroles multiples. Il s'agit d'une science qui a su, de par ses interrogations et découvertes, attirer l'intérêt du grand public, d'une science qui a ses vedettes, qu'elles soient parmi les primates (le cas de Lucy est de loin le plus frappant) ou parmi les scientifiques qui se sont chargés de sa sociodiffusion ( $Y$. Coppens précise dans un article de

1 C. Belisle, "Enjeux et limites du multimédia en formation et en éducation", Les cahiers de l'Asdifle, Actes des 19e et 20e rencontres, Paris, 1998, p. 18. 
juillet 1993 avoir derrière lui "quelque 300 à 400 apparitions à la télévision et pas moins de 600 à 700 émissions de radio"1).

La paléontologie marine, quant à elle, est une discipline moins médiatisée. Les articles qui lui sont consacrés, beaucoup plus courts (parfois des dépêches de l'AFP ou de Reuter) sont souvent publiés à l'occasion de la découverte de nouveaux fossiles ou font écho à des publications figurant dans Nature. Ils usent du même appareil descriptif que celui convoqué dans le cédérom et affichent de temps à autres le même souci de présenter leur objet dans ses aspects les plus proches de l'homme. A cette image discontinue et limitée parfois à de gros titres sur la découverte d'un fossile, le cédérom propose une image de la science dans toute sa richesse sans pour autant que la modernité des objets convoqués par sa scénographie (aquarium virtuel, paléoscaphe, laboratoire des évolutions, œuf généalogique) ne pallie son mode expositif, détaché de l'individu et sa représentation muséographique quelque peu poussiéreuse.

Si nous partons du principe que la mise en cédérom est une bonne occasion pour présenter un sujet, une thématique ou, ici, un savoir disciplinaire, "autrement" nous remarquerons toutefois que les enjeux du numérique ne semblent pas être les mêmes pour la paléontologie et pour la paléoanthropologie. Toutes deux proposent bien sûr une synthèse en la matière ; mais cet objectif général commun est mis a priori au service de deux stratégies bien distinctes. Il s'agit pour la paléontologie de proposer en priorité une image relativement complète du savoir, compensant d'une certaine manière sa diffusion limitée auprès du grand public. En revanche, dans le cas de la paléoanthropologie, l'effet-synthèse semble devenir, entre autres, une sorte de prétexte pour faire l'apologie ${ }^{2} \mathrm{~d}^{\prime}$ une science souvent présentée dans la presse comme perplexe, incertaine, contradictoire, condamnée “à n'avancer qu'à tâtons dans la connaissance de notre passé"3.

I Le Monde, "Eurêka! J'ai tout faux", 5 juillet 1993, p. 21.

${ }^{2}$ L'entrée Une expédition archéologique dans Aux origines de l'homme est, sur ce point, particulièrement caractéristique. Elle simule, comme nous l'avons vu, sous prétexte d'un jeu, le travail sur le terrain, définit les différentes spécialités auxquelles fait appel la paléoanthropologie et les démarches dont elles usent pour coordonner l'ensemble du travail et le mener à bien. L'image ici proposée est celle d'une science plurielle dans ses compétences, unie, établissant ses calculs de manière précise et rigoureuse sans que sa mise en verbe ne le soit nécessairement.

3 Le Monde, "Histoires de famille", 24 novembre 1993, p. 15. 